\title{
Observation of optical precursors at the biphoton level
}

\author{
Shengwang Du, Chinmay Belthangady, Pavel Kolchin, G. Y. Yin, and S. E. Harris \\ Edward L. Ginzton Laboratory, Stanford University, Stanford, California 94305, USA \\ *Corresponding author: shengwang.du@gmail.com
}

\begin{abstract}
Compiled July 25, 2008
We describe the observation of a sharp leading edge spike in a biphoton wave packet that is produced using slow light and measured by two photon correlation. Using the stationary phase approximation we characterize this spike as a Sommerfeld-Brillouin precursor resulting from the interference of low and high frequency spectral components. (c) 2008 Optical Society of America OCIS codes: $270.0270,070.7345$.
\end{abstract}

Optical precursors were first described by Sommerfeld and Brillouin in 1907, and are of importance in electromagnetic theory because they ensure that the front edge of a wave packet will always travel at the velocity of light in vacuum. It was previously believed that optical precursors existed for only a few optical cycles and contributed little to the amplitude of the field [1]. However, recent theoretical [2] and experimental [3] work has shown that in a narrow-resonance atomic medium the precursor magnitude can be of the same order as the primary field and its length may be much longer than a few cycles. Precursors have been extensively studied in the gamma ray [4], microwave [5], and optical regimes $[3,6-9]$.

In this Letter, we extend the classical concept of precursors to describe the behavior of biphoton wavepackets that are measured by correlation using single photon counting modules (SPCM). The measured wave packets are generated by using electromagnetically induced transparency (EIT) and slow light $[10,11]$ so that the correlation time of the main body of the wave packet can be varied over the range of $50-900$ ns [12]. A requirement for the sharp front edge spike to be apparent is that the optical depth (OD) be sufficiently large that the pulse length is determined by the group delay. We remark that the observation of the sharp leading edge spike, see Fig. 2(b), was not expected, and its nature was first suggested to us by Daniel J. Gauthier.

A schematic of biphoton generation is shown in Fig. 1. In the presence of counter-propagating cw pump $\left(\omega_{p}\right)$ and coupling $\left(\omega_{c}\right)$ lasers, phase-matched, paired Stokes $\left(\omega_{s}\right)$ and anti-Stokes $\left(\omega_{a s}\right)$ photons are spontaneously generated and propagate in opposite directions. We use a two-dimensional (2D) ${ }^{85} \mathrm{Rb}$ magneto-optical trap (MOT) with a longitudinal length $L=1.7 \mathrm{~cm}$ and an aspect ratio of 25 . The Stokes $\left(\sigma^{-}\right)$and anti-Stokes $\left(\sigma^{+}\right)$ photons are coupled into opposing single mode fibers, and detected by two SPCMs after passage through two narrow-band optical filters (F1 and F2). With the dephasing rate of the $|1\rangle \rightarrow|3\rangle$ transition denoted by $\gamma_{13}=2 \pi \times 3 \times 10^{6} \mathrm{sec}^{-1}$, the pump laser has a Rabi frequency of $\Omega_{p}=1.16 \gamma_{13}$, is $\sigma^{-}$circularly polarized, and is blue detuned from the $|1\rangle \rightarrow|4\rangle$ transition by

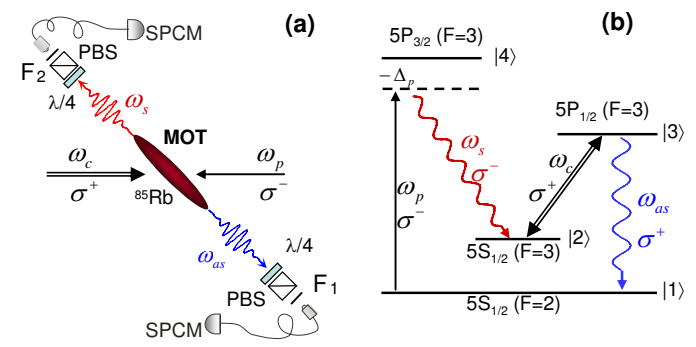

Fig. 1. (color online). Biphoton generation in a double- $\Lambda$ system. (a) Experimental configuration. (b) ${ }^{85} \mathrm{Rb}$ energy level diagram; (from Ref. [12]).
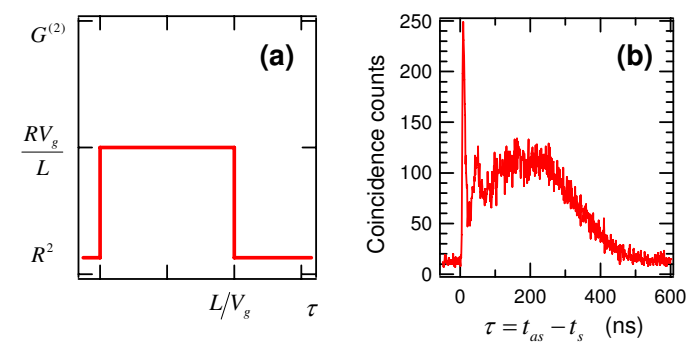

Fig. 2. (color online). (a) Two-photon correlation for an ideal spontaneous parametric down converter with a relative group delay $L / V_{g}$ and paired emission rate $R$. (b) Experimental Stokes-anti-Stokes coincidence counts per $1 \mathrm{~ns}$ bin in $800 \mathrm{~s}$ as a function of delay at an optical depth of $62, \Omega_{c}=4.20 \gamma_{13}, \Omega_{p}=1.16 \gamma_{13}$, and $\Delta_{p}=48.67 \gamma_{13}$.

$\Delta_{p}=48.67 \gamma_{13}$. The coupling laser $\left(\Omega_{c}\right)$ is $\sigma^{+}$circularly polarized, and is on resonance with the $|2\rangle \rightarrow|3\rangle$ transition. Further experimental details are described in [12].

We first note that the Glauber correlation function, or equivalently the coincidence rate versus the relative time delay $\tau$ for an ideal parametric down converter with a frequency independent group delay between the signal and idler of $L / V_{g}$ is a rectangle [Fig. 2(a)]. Each biphoton (pair of photons) is emitted at a random position; if the point of emission is at the front edge of the nonlinear medium, then the photons arrive simultaneously; if it is at the back edge, then one is delayed from the 


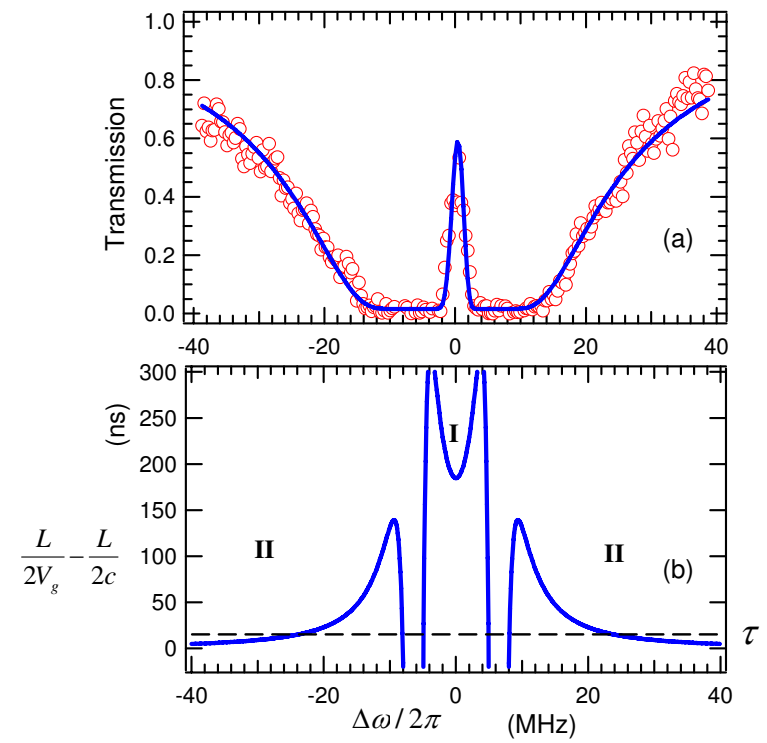

Fig. 3. (color online). (a)Anti-Stokes (EIT) transmission profile. (b) Group delay $L / 2 V_{g}$ as a function of antiStokes detuning $\Delta \omega$. Optical depth $=62, \Omega_{c}=4.20 \gamma_{13}$.

other by $L / V_{g}$. For an EIT-based down converter operating at high optical depth, the anti-Stokes photon travels slowly and because the bandwidth is constrained by the EIT profile, the correlation profile is smoothed and has a full width equal to the group delay. Figure 2 (b) shows the experimentally observed correlation function with a correlation width of about $400 \mathrm{~ns}$ as compared to the measured group delay of $L / V_{g}=372 \mathrm{~ns}$. It is the sharp peak and the oscillatory structure at the leading edge that require further explanation.

The experimental curve, including the leading edge, is in good agreement with the Heisenberg picture theory of earlier papers from our group [12-14]. To explicitly display the phase of the biphoton, we start here with the equivalent Schrödinger picture wave function. With the assumption that the off-resonant pumping laser is sufficiently weak that the atomic population remains primarily in the ground level $|1\rangle$, and $\tau=t_{a s}-t_{s}$, the two time Stokes-anti-Stokes biphoton wave function is $\Psi\left(t_{s}, t_{s}+\tau\right)=\psi(\tau) e^{-i\left(\omega_{c}+\omega_{p}\right) t_{s}}$, where the envelope quantity $[15-17]$

$$
\psi(\tau)=\frac{L}{2 \pi} \int \kappa(\omega) \phi(\omega) e^{i \theta(\omega, \tau)} d \omega
$$

Here the quantity $\omega$ denotes the anti-Stokes frequency, $\kappa(\omega)$ is the nonlinear coupling coefficient, and $\theta(\omega, \tau)=$ $\operatorname{Re}\left[\left(k_{a s}+k_{s}\right) L / 2-\omega \tau\right]$ is the real phase function. $k_{a s}(\omega)$ and $k_{s}(\omega)$ are the complex angular wavenumbers. With $\Delta k(\omega)=\left(\vec{k}_{a s}-\vec{k}_{s}^{*}-\vec{k}_{c}+\vec{k}_{p}\right) \cdot \vec{z}$, and the unit vector $\vec{z}$ taken in the direction of anti-Stokes generation, $\phi(\omega)=$ $\operatorname{sinc}(\Delta k L / 2) \exp \left\{-\operatorname{Im}\left[\left(k_{a s}+k_{s}\right) L / 2\right]\right\}$. Expressions for these quantities in terms of the pertinent susceptibilities are given in [17]. The two-photon Glauber correlation function can be expressed in terms of the biphoton wave function as: $G^{(2)}(\tau)=|\psi(\tau)|^{2}+B$, where the background $B$ results from uncorrelated photons.

As is the case for propagating classical wave packets, the method of stationary phase may be used to explain the biphoton precursor. The essential idea is that the dominant contribution to the integral in Eq. (1) occurs when the derivative of $\theta(\omega, \tau)$ is equal to zero [18]. For different frequencies this zero occurs at different times $\tau$. By differentiating $\theta(\omega, \tau)$ we see that the relative delay time $\tau$ is related to the dominant frequency $\omega_{d}$ by

$$
\tau=\frac{L}{2 V_{g}\left(\omega_{d}\right)}-\frac{L}{2 c},
$$

where we have taken $k_{s}=\left(\omega_{c}+\omega_{p}-\omega\right) / c$.

The upper and lower portions of Fig. 3 show the measured EIT transmission and the calculated right hand side of Eq. (2) as functions of anti-Stokes frequency detuning $\Delta \omega=\omega-\omega_{13}$ from $|1\rangle \rightarrow|3\rangle$ transition, respectively. Though it is not apparent in this figure, the rightside asymptote approaches zero faster than does the left asymptote. Therefore the earliest portion of the biphoton wave packet to intersect with the horizontal line $\tau$ and to arrive at the detector comes from the high frequency portion of the spectrum and is thought of as the Sommerfeld precursor. At slightly later times the low frequency Brillouin components arrive at the detector and beat with the simultaneously arriving high frequency components.

We may think of $\psi(\tau)$ in Eq. (1) as having two portions that arise from different regions in frequency space, i.e.

$$
\psi(\tau)=\psi_{0}(\tau)+\psi_{S B}(\tau) .
$$

The function $\psi_{0}(\tau)$ is the main body of the biphoton and contains most of its energy. It is obtained by numerically integrating over the central region I of Fig. 3 that extends $\Delta \omega$ from $-\Omega_{c} / 2$ to $\Omega_{c} / 2$ :

$$
\psi_{0}(\tau)=\frac{L}{2 \pi} \int_{\omega_{13}-\Omega_{c} / 2}^{\omega_{13}+\Omega_{c} / 2} \kappa(\omega) \phi(\omega) e^{i \theta(\omega, \tau)} d \omega .
$$

The function $\psi_{S B}(\tau)$ is the Sommerfeld-Brillouin precursor and is obtained by integrating over region II of Fig. 3. For an oscillatory integral of the form $f=$ $\int_{-\infty}^{\infty} F(\omega) e^{i \theta(\omega)} d \omega$ where $F(\omega)$ varies slowly as compared to the real phase $\theta(\omega)$, the dominant contribution to the integral occurs when the derivative, $\theta^{\prime}\left(\omega_{d}\right)$ is equal to zero. We expand $\theta(\omega) \simeq \theta\left(\omega_{d}\right)+\frac{1}{2} \theta^{\prime \prime}\left(\omega_{d}\right)\left(\omega-\omega_{d}\right)^{2}$ and integrate over $\omega$. With the contribution of the end points neglected, this integral is $f_{d}=\sqrt{\frac{2 \pi}{-i \theta^{\prime \prime}\left(\omega_{d}\right)}} F\left(\omega_{d}\right) e^{i \theta\left(\omega_{d}\right)}$. When there are several points of stationary phase, it follows that the Sommerfeld-Brillouin portion of the wave packet is [18]

$$
\psi_{S B}(\tau)=\sum_{\omega_{d}} \frac{\kappa\left(\omega_{d}\right) \phi\left(\omega_{d}\right) L}{\sqrt{-i 2 \pi \theta^{\prime \prime}\left(\omega_{d}\right)}} e^{i \theta\left(\omega_{d}, \tau\right)} .
$$

Figure 4 is a zoomed view of Fig. 2(b) from 0 to 125 ns. The (blue) theoretical curve is obtained from Eqs. (3)-(5) 


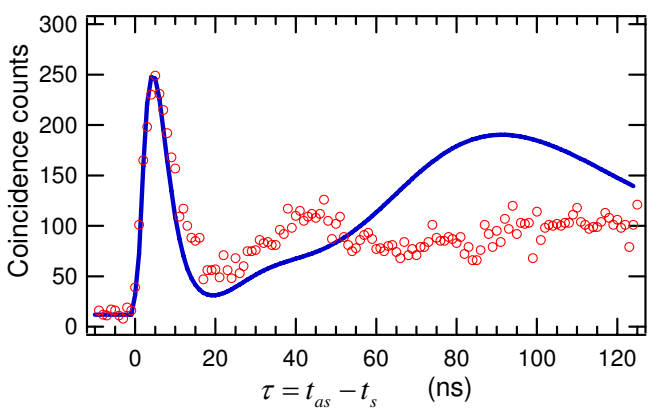

Fig. 4. (color online). Zoomed view of Fig. 2(b) comparing the experimental data (circles, red) and the theoretical curve (solid line, blue). Optical depth $=62$.

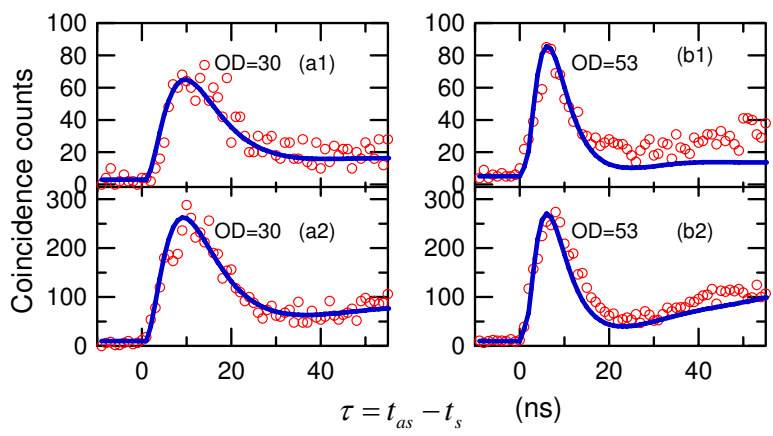

Fig. 5. (color online). Comparison of theory and experiment for other paratmeters. In parts (a1) and (b1) $\Omega_{c}=2.35 \gamma_{13}$. In parts (a2) and (b2) $\Omega_{c}=4.20 \gamma_{13}$.

with a vertical scaling factor to best fit the experimental data. The leading edge Sommerfeld-Brillouin precursor has a width of about $10 \mathrm{~ns}$. Experimental and theoretical results at smaller OD (30 and 53) and different $\Omega_{c}$ are plotted in Fig. 5. All show reasonable agreement between Eq. (1), or equivalently Eqs. (3)-(5) and experiment.

The width at half power of the precursor as determined by evaluating Eq. (5) is $7 \mathrm{~ns}$ at an optical depth of 62. A still coarser estimate is obtained by taking the functional form of the beating as $\cos ^{2}\left(\Delta \omega_{d} \tau\right)$ with the width at half power occuring when $\Delta \omega_{d} \tau=\pi / 4$, and the line shape as Lorentzian. The group delay at a detuning $\Delta \omega$ in the wing of a naturally broadened Lorentzian line may be expressed in terms of the Einstein A coefficient and the optical depth $N \sigma L$ as $L / V_{g}=A N \sigma L /\left(4 \Delta \omega^{2}\right)$, where $N$ is atomic density and $\sigma$ is the anti-Stokes onresonance absorption cross section. Combining these expressions, the half power width of the precursor spike is $\pi^{2} /(A N \sigma L)$. At an optical depth of 62 as in Fig. (4), and a natural decay time of $26.5 \mathrm{~ns}\left(A=2 \gamma_{13}\right)$, this formula predicts a precursor width of $4.2 \mathrm{~ns}$.

It might be hypothesized that the observed shape is not a Sommerfeld-Brillouin precursor, but instead results from the high optical depth and non-zero loss experienced by the anti-Stokes field. To test this we have used Eq. (1) and also the exact operator equations with, in both cases, the spontaneous decay rate and both dephasing rates set to zero, thereby creating a lossless system. We find that except for a slightly more pronounced oscillation, the precursor has the same shape as in Figs. 4 and 5. We have thereby verified that the SommerfeldBrillouin precursor is dominantly the result of the interplay of phases during nominally lossless propagation.

The authors thank D. J. Gauthier, J. -M. Wen, and H. Jeong for helpful discussions. The work was supported by the Defense Advanced Research Projects Agency, the U.S. Air Force Office of Scientific Research, and the U.S. Army Research Office.

S. Du's e-mail address is shengwang.du@gmail.com. S. Du's present address is Department of Physics, The Hong Kong University of Science and Technology, Hong Kong, China.

\section{References}

1. L. Brillouin, Wave Propagation and Group Velocity (Academic Press, New York 1960).

2. W. R. LeFew, S. Venakides, and D. J. Gauthier, arXiv:0705.4238 [physics.optics].

3. H. Jeong, A. M.C. Dawes, and D. J. Gauthier, Phys. Rev. Lett. 96, 143901 (2006).

4. F. J. Lynch, R. E. Holland, and M. Hamermesh, Phys. Rev. 120, 513 (1960).

5. P. Pleshko and I. Palocz, Phys. Rev. Lett. 22, 1201 (1969).

6. J. Aavikscoo, J. Lippmaa, and J Kuhl, J. Opt. Soc. Am. B 5, 1631 (1988).

7. J. Aavikscoo, J Kuhl, and K Ploog, Phys. Rev. A 44, R5353 (1991).

8. S.-H. Choi and U. L. Österberg, Phys. Rev. Lett. 92, 193903 (2004).

9. H. Jeong and U. L. Österberg, Phys. Rev. A 77, 021803(R) (2008).

10. S. E. Harris, Phys. Today 50 (7), 36 (1997).

11. M. Fleischhauer, A. Imamoglu, and J. P. Marangos, Rev. Mod. Phys. 77, 633 (2005).

12. S. Du, P. Kolchin, C. Belthangady, G. Y. Yin, and S. E. Harris, Phys. Rev. Lett. 100, 183603 (2008).

13. V. Balić, D. A. Braje, P. Kolchin, G.Y. Yin, and S. E. Harris, Phys. Rev. Lett. 94, 183601 (2005).

14. Pavel Kolchin, Phys. Rev. A 75, 033814 (2007).

15. M. H. Rubin, D. N. Klyshko, Y. H. Shih, and A. V. Sergienko, Phys. Rev. A 50, 5122 (1994).

16. S. Du, E. Oh, J.-M Wen, and M. H. Rubin, Phys. Rev. A 76, 013803 (2007).

17. S. Du, J.-M Wen, and M. H. Rubin, J. Opt. Soc. Am. B (accepted 2008); arXiv:0804.3981 [quant-ph].

18. J. D. Jackson, Classical Electrodynamics, 2nd ed. (Wiley, New York, 1975). 